Les lasers et leurs applications scientifiques et médicales

\title{
Bruit quantique dans les lasers
}

\author{
C. Fabre
}

Laboratoire Kastler-Brossel, Université Pierre et Marie Curie, Case 74, 75252 Paris cedex 05, France

\begin{abstract}
Dans de nombreuses applications, les problèmes de stabilité de fréquence et d'intensité du rayonnement laser jouent un rôle crucial. Le "bruit " incontrôlable de la source constitue en effet une limitation des performances du système. L'exemple extrême en la matière est fourni par les détecteurs interférométriques de rayonnement gravitationnel (voir cours de C. Nary Man), où en raison de la faiblesse extrême du signal à mesurer, la fréquence et l'intensité de la source laser doivent atteindre une extraordinaire stabilité. Pour l'essentiel, le bruit dans les lasers est d'origine "technique ", c'est-à-dire qu'il est lié à des fluctuations difficilement maîtrisables de divers paramètres du laser (longueur de la cavité, nombre d'éléments actifs dans l'amplificateur laser, taux de pompage...). Comme le montre le cours de C. Chardonnet, ces bruits peuvent être diminués, au prix de beaucoup de travail et d'astuces technologiques. Mais il est utile de savoir si ces efforts sont appelés à se heurter tôt ou tard à des limites imposées par la physique même du processus, ou si on peut espérer faire tendre ces bruits vers zéro. L'objet de ce cours est d'exposer les idées de base sur les limites imposées par la mécanique quantique au bruit d'un faisceau laser. Nous commencerons par quelques rappels relatifs au bruit quantique d'un faisceau lumineux libre (section 1), puis nous traiterons des bruits quantiques dans l'amplification optique (section 2) et dans le laser (section 3). Nous terminerons dans la section 4 par une description des résultats expérimentaux relatifs aux " lasers sub-Poissoniens », c'est-à-dire ayant un bruit d'intensité inférieur au bruit de grenaille ou « shot noise ».
\end{abstract}

\section{FLUCTUATIONS QUANTIQUES DE LA LUMIÈRE}

Nous rappelons ici brièvement les caractéristiques essentielles des fluctuations d'origine quantique du champ électromagnétique. On trouvera dans les références [1-4] un exposé beaucoup plus détaillé des propriétés quantiques de la lumière.

\subsection{Cas d'un mode unique du champ}

Considérons un mode du champ défini par sa fréquence $\omega$, son vecteur d'onde $\mathbf{k}$ et sa polarisation supposée rectiligne $\varepsilon$ (un laser monomode peut être considéré comme émettant un tel mode, si on ne s'intéresse pas aux effets liés à la variation transverse d'intensité). 


\subsubsection{Description classique}

En tout point $\mathbf{r}$ et à chaque instant $t$ le champ électrique de cette onde s'écrit :

$$
\begin{aligned}
\mathbf{E}(\mathbf{r}, t) & =E_{0} \varepsilon \cos (\omega t-\mathbf{k} \cdot \mathbf{r}+\varphi) \\
& =\varepsilon\left(E_{1} \cos (\omega t-\mathbf{k} \cdot \mathbf{r})+E_{2} \sin (\omega t-\mathbf{k} \cdot \mathbf{r})\right)
\end{aligned}
$$

Le mode est donc défini par la donnée de deux paramètres réels : amplitude et phase $\left(E_{0}, \varphi\right)$ ou bien les deux « composantes de quadrature » $E_{1}, E_{2}$. Il est aussi défini par la donnée d'un point $\mathrm{M}$ dans un espace à deux dimensions (Figure 1a), appelé « plan de Fresnel » dont $\left(E_{0}, \varphi\right)$ et $\left(E_{1} E_{2}\right)$ sont respectivement les coordonnées polaires ou cartésiennes. L'évolution temporelle, ou spatiale le long de la direction de propagation, de ce mode correspond alors simplement à une rotation du point $\mathrm{M}$ autour de l'origine dans ce plan.

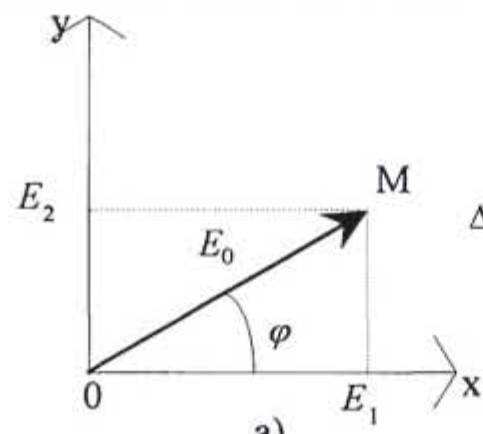

a)

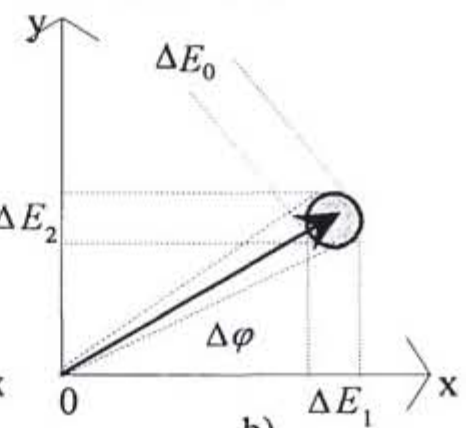

b)

Figure 1

\subsubsection{Description quantique : opérateur champ}

La théorie quantique des champs impose le passage d'une description en termes de fonctions réelles, ou complexes, de $t$ et de $\mathbf{r}$ à une description en termes d'opérateurs et de fonctions d'onde. Il s'agit de la même transposition que dans le cas, plus connu, de la position $x(t)$ et l'impulsion $p(t)$ d'une particule matérielle classique, que l'on remplace en théorie quantique par des opérateurs $\hat{x}$ et $\hat{p}$ qui ne commutent pas. Le champ électrique, en représentation de Heisenberg, est alors associé à un opérateur $\hat{E}(\mathbf{r}, t)$ donné par :

$$
\hat{E}(r, t)=\vec{\varepsilon}\left(\hat{E}_{1} \cos (\omega t-k \cdot r)+\hat{E}_{2} \sin (\omega t-k \cdot r)\right)
$$

(le symbole ${ }^{\wedge}$ signale la présence d'un opérateur quantique)

$\hat{E}_{1}$ et $\hat{E}_{2}$ sont des opérateurs hermitiens qui s'expriment en fonction des opérateurs de création $\left(\hat{a}^{+}\right)$et d'annihilation $(\hat{a})$ de photon dans le mode considéré sous la forme :

$$
\hat{\mathrm{E}}_{1}=\mathrm{E}_{\omega}\left(\hat{\mathrm{a}}+\hat{\mathrm{a}}^{+}\right) \quad \hat{\mathrm{E}}_{2}=\frac{1}{\mathrm{i}} \mathrm{E}_{\omega}\left(\hat{\mathrm{a}}-\hat{\mathrm{a}}^{+}\right) \quad\left[\hat{\mathrm{a}}, \hat{a}^{+}\right]=1
$$

avec $E_{\omega}^{2}=\left(\hbar \omega / 2 \varepsilon_{0} V\right)^{1 / 2}(V$ : volume de quantification) 
Les opérateurs de quadrature $\hat{E}_{1}$ et $\hat{E}_{2}$ ne commutent pas. D'après (3), on a, de la même manière que $[\hat{x}, \hat{p}]=\mathrm{i} \hbar$ :

$$
\left[\hat{E}_{1}, \hat{E}_{2}\right]=2 i E_{\omega}^{2}
$$

La détermination exacte du mode du champ requiert la mesure simultanée des variables $E_{l}$ et $E_{2}$. La relation de non-commutation (4) empêche que cette mesure simultanée se fasse avec une précision absolue sur les deux grandeurs. Il va en résulter une dispersion sur la détermination de la position de $\mathrm{M}$, que l'on peut symboliser par la surface grisée de la figure 1b. L'extension de cette zone d'indétermination est caractérisée par les écarts quadratiques moyens $\Delta E_{1}$ et $\Delta E_{2}$, qui satisfont à l'inégalité de Heisenberg que l'on déduit aisément de (4) :

$$
\Delta E_{1} \Delta E_{2} \geq E_{\omega}^{2}
$$

La surface de la zone d'indétermination a donc une aire minimale égale à $E_{\text {c }}^{2}$. On a évidemment aussi dispersion des résultats si on mesure l'amplitude $E_{0}$ et la phase $\varphi$ du champ, qui se traduit par les inégalités :

$$
\Delta E_{0} \Delta \varphi \geq E_{\omega}^{2} /\left\langle E_{0}\right\rangle \quad \Delta N \Delta \varphi \geq \frac{1}{2}
$$

(valable pour des champs dans lesquels le nombre moyen de photons est grand devant 1), $N$ est la mesure du nombre de photons dans le mode, associé à l'opérateur quantique $\hat{N}=\hat{a}^{+} \hat{a}$, et aussi proportionnel au carré de l'amplitude $E_{0}^{2}$. La dernière inégalité illustre bien l'incompatibilité - ou la complémentarité - entre la description corpusculaire (en termes de nombre de photons) et la description ondulatoire (où la phase $\varphi$ du champ joue un rôle déterminant) du champ électromagnétique.

\subsubsection{Description quantique : états possibles du champ}

Les opérateurs associés aux mesures étant définis, il faut maintenant s'intéresser aux états possibles du champ, décrits par des vecteurs d'états. Passons en revue quelques états particulièrement importants.

a) le " vide " $|0\rangle$ (figure 2a) : c'est l'état fondamental du champ dans le mode considéré, que l'on pourrait dénommer de manière plus appropriée "obscurité ». Il correspond à un champ nul en valeur moyenne, mais non pas en variances, car $\Delta E_{1}=\Delta E_{2}=0$ est contradictoire avec la relation (5). Il est ainsi le siège de fluctuations non nulles autour de 0 :

$$
\Delta E_{1}=\Delta E_{2}=E_{\infty}
$$

$E_{\omega}$ représente donc l'amplitude des "fluctuations du vide», qui présentent bien des analogies avec les «fluctuations de point zéro » des oscillateurs harmoniques matériels comme par exemple les vibrations d'un cristal.

b) l'état cohérent $|\alpha\rangle$ (figure $2 b$ ) (ou " état quasi classique ») défini comme état propre de l'opérateur $\hat{a}: \hat{a}|\alpha\rangle=\alpha|\alpha\rangle$. On montre facilement qu'il correspond à un champ non nul en valeur moyenne, repéré dans le plan de Fresnel par le nombre complexe $2 E_{\omega} \alpha$, avec des fluctuations identiques à celles du vide (éq. 7), c'est-à-dire minimales et identiques sur les deux quadratures. Il en résulte des fluctuations sur l'amplitude, le nombre de photons et la phase, données, lorsque $|\alpha| \gg 1$, par : 


$$
\Delta E_{0}=E_{\omega}, \quad \Delta N=|\alpha|=\sqrt{\langle\hat{N}\rangle} \quad \Delta \varphi=\frac{1}{2|\alpha|}=\frac{1}{2 \sqrt{\langle\hat{N}\rangle}}
$$

où $\langle\hat{N}\rangle=|\alpha|^{2}$ est le nombre moyen de photons dans l'état cohérent.

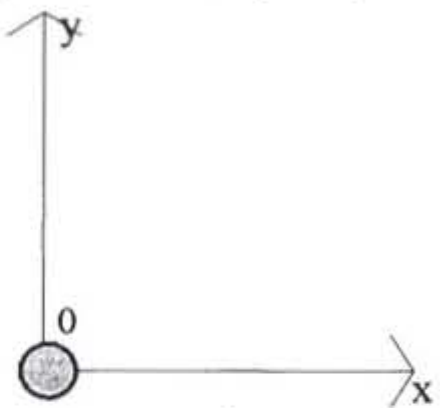

a)

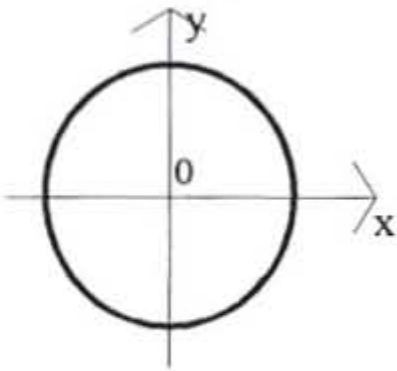

c)

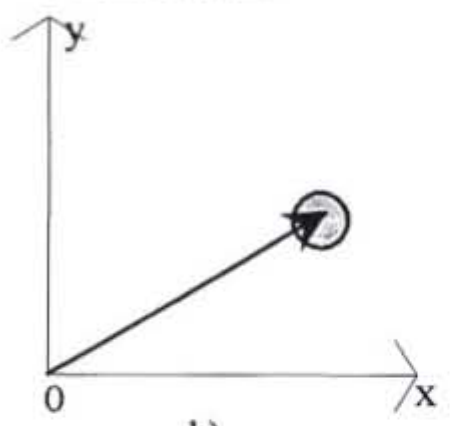

b)

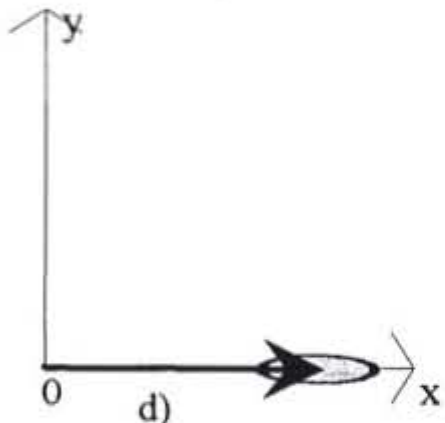

d)

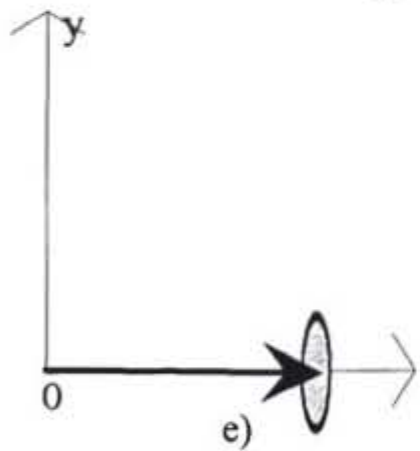

Figure 2

On appellera $\sqrt{\langle\hat{N}\rangle}=\Delta N_{s T}, \frac{1}{2 \sqrt{\langle\hat{N}\rangle}}=\Delta \varphi_{S T}$ les « bruits quantiques standard " d'intensité et de phase. La probabilité $P(n)$ de mesurer un nombre de photons $n$ dans un tel état vaut : $P(n)=e^{-|\alpha|^{2}}|\alpha|^{2 n} / n !$ : c'est une distribution de Poisson, analogue à ce qu'on obtiendrait si le 
faisceau était composé de particules ponctuelles (les photons) statistiquement indépendantes les unes des autres. On comprend alors pourquoi le bruit d'intensité d'un tel faisceau est appelé bruit de grenaille, ou " shot noise ».

c) l'état nombre $|n\rangle$ (figure 2c), défini comme état propre de $\hat{N}=\hat{a}^{+} \hat{a}: \quad \hat{N}|n\rangle=n|n\rangle$. C'est un état propre de l'énergie du champ. Il est caractérisé par les variances :

$$
\Delta E_{1}=\Delta E_{2}=E_{\omega} \sqrt{2 n+1} \quad \Delta N=0 \quad \Delta E_{0}=0 \quad \Delta \varphi=\infty
$$

Ce n'est donc pas un état minimal vis-à-vis de la relation de Heisenberg (5). En revanche, il permet des mesures d'intensité ou d'amplitude sans aucune incertitude. Le prix à payer est l'absence totale d'information de phase dans un tel état.

d) les états comprimés (ou squeezés). Ils sont étudiés en détail dans les références [1-4]. Ce sont des états minimaux vis-à-vis de la relation (5) mais dans lesquels il n'y a plus égalité entre $\Delta E_{1}$ et $\Delta E_{2}$. Sur la figure $2 \mathrm{~d}$, on a représenté un état tel que $\Delta E_{1}>E_{\omega}$ et $\Delta \mathrm{E}_{2}<E_{\omega}$. Dans un tel état, la phase est mieux définie que dans un état cohérent : $\Delta \varphi<\Delta \varphi_{S T}$. Mais on a alors $\Delta N>\sqrt{\langle\hat{N}\rangle}$. Inversement, sur la figure $2 \mathrm{e}$, on a un état comprimé tel que $\Delta E_{1}<E_{\omega} \quad \Delta E_{2}>E_{\omega}$. L'intensité y présente alors moins de fluctuations que dans un état cohérent $\Delta N<\sqrt{\langle\hat{N}\rangle}$. Le bruit de phase est alors augmenté : $\Delta \varphi>\Delta \varphi_{s t}$. On qualifie souvent un tel état, pour des raisons évidentes, d'état « sub-Poissonien ».

Parmi tous les états du champ que nous venons d'énumérer, il y en a de plus ou moins " exotiques». Les état (a) et (b) peuvent être produits par les sources habituelles ou sources " classiques » de lumière. Il n'en est pas de même des états (c), (d) ou (e), dont la production nécessite des processus non linéaires complexes et qui sont fragiles vis-à-vis des pertes. Les états nombres en particulier n'ont jamais été réalisés en pratique pour $n \geq 2$. Les états comprimés n'ont été produits en laboratoire que depuis 1985 [5]. Les états tels que (c), (d) et (e) sont ainsi qualifiés de « non-classiques ».

D'une manière plus formelle, nous appellerons «états classiques » des états de la lumière tels que $\Delta E_{1}=\Delta E_{2}$. Pour de tels états, la relation de Heisenberg (5) sur le produit des variances se transforme en inégalité sur chacune d'entre elles :

$$
\Delta E_{1}=\Delta E_{2}: \Delta E_{1}>E_{\omega} \quad \Delta E_{2}>E_{\omega}
$$

Donc pour de tels états classiques :

$$
\Delta E_{0}>E_{\omega} \quad \Delta N \geq \Delta N_{S T}=\sqrt{\langle\hat{N}\rangle} \quad \Delta \varphi \geq \Delta \varphi_{S T}=\frac{1}{2 \sqrt{\langle\hat{N}\rangle}}
$$

De tels états, les plus faciles à produire, ont donc des fluctuations d'amplitude, de nombre de photons ou de phase limités inférieurement par les «limites quantiques standard » données par les relations (8). Ces limites, qui ne sont que des «limites de commodité », relatives à un sous-ensemble d'états possibles du champ, ont longtemps été considérées comme une limite infranchissable en optique.

\subsection{Cas d'un faisceau lumineux non monochromatique}

Considérons, de manière plus réaliste que dans le paragraphe précédent, un faisceau lumineux, émis par exemple par un laser, de polarisation $\varepsilon$, ayant une dispersion en fréquence autour d'une fréquence moyenne $\omega_{0}$ et une extension latérale caractérisée par une surface $S$. Plaçons 
un détecteur sur ce faisceau ; le champ électrique de l'onde au centre du faisceau et sur le détecteur peut s'écrire :

$$
\mathbf{E}(t)=\varepsilon\left(E_{1}(t) \cos \omega_{0} t+E_{2}(t) \sin \omega_{0} t\right)
$$

Le faisceau n'étant pas exactement monochromatique, les composantes de quadrature dépendent maintenant du temps (avec des fréquences caractéristiques supposées cependant très petites devant $\omega_{0}$ ).

On associe à ces grandeurs classiques des opérateurs quantiques : $\hat{E}_{1}(t), \hat{E}_{2}(t)$ dont on peut montrer qu'ils satisfont à la relation de commutation suivante :

$$
\left[\hat{E}_{1}(t), \hat{E}_{2}\left(t^{\prime}\right)\right]=i \frac{\hbar \omega_{0}}{\varepsilon_{0} c S} \delta\left(t-t^{\prime}\right)
$$

La mesure du champ sur le détecteur va être affectée d'un bruit caractérisé par une variance dont la valeur dépend de la bande passante en fréquence $\delta f$ de l'amplificateur utilisé pour la mesure. On obtient ici aussi à partir de (13) une relation de Heisenberg qui s'écrit :

$$
\Delta E_{1} \Delta E_{2} \geq \frac{\hbar \omega_{0}}{4 \varepsilon_{0} c S} \delta f
$$

Les détecteurs existant à l'heure actuelle dans le domaine optique ne sont pas assez rapides pour réagir à l'oscillation de l'onde à la fréquence $\omega_{0}$. Ils ne mesurent que sa puissance moyenne (moyennée sur un grand nombre de périodes optiques) exprimée en watts, $\mathrm{P}=\frac{1}{2} \varepsilon_{0} \mathrm{cS}\left(\mathrm{E}_{1}^{2}+\mathrm{E}_{2}^{2}\right)$. Cette puissance peut ensuite être transcrite en termes de débit numérique I de photons. $\mathrm{Si}$ on considère un détecteur de rendement quantique unité (il en existe dans la pratique), on a :

$$
I=\frac{\mathrm{P}}{\hbar \omega_{0}}
$$

De (14), on déduit des relations relatives aux bruits $\Delta \mathrm{P}, \Delta l$ et $\Delta \phi$ existant respectivement sur la puissance $\mathrm{P}$, le débit de photons $I$ et la phase $\varphi$ du faisceau lumineux :

$$
\Delta \mathrm{P} \Delta \varphi \geq \hbar \omega_{0} \delta f \quad \Delta I \Delta \varphi \geq \delta f
$$

Il existe une manière plus précise de caractériser le bruit d'un signal $x(t)$ en physique, qui a en outre l'avantage d'être indépendante de la bande passante $\delta f$ de l'appareil de mesure utilisé : il s'agit de la densité spectrale du bruit $S_{x}(\Omega),(1)$ qui est la transformée de Fourier de la fonction d'autocorrélation de $x(t)$ :

$$
\mathrm{S}_{\mathrm{x}}(\Omega)=\int_{-\infty}^{+\infty} \mathrm{dt}\langle\mathrm{x}(0) \mathrm{x}(\mathrm{t})\rangle \mathrm{e}^{i \Omega \mathrm{t}}
$$

Une telle définition se transpose aisément pour des observables quantiques. On déduit alors de (13) des relations de Heisenberg relatives aux densités spectrales de bruit. En particulier pour le débit de photons $I$ et la phase $\varphi$, on obtient une relation proche de (6) :

(1) Si on mesure le signal $x$ à l'aide d'un filtre passe-bande laissant passer les fréquences dans l'intervalle $\left[f_{0}-\delta f / 2, f_{0}+\delta / 2\right]$, on observera un bruit dont l'écart quadratique moyen est relié à la densité spectrale de bruit par la relation : $\Delta x=\sqrt{2 S_{x}\left(\Omega=2 \pi f_{0}\right) \delta f}$. 


$$
S_{/}(\Omega) S_{\varphi}(\Omega) \geq \frac{1}{4}
$$

Cette limite est valable quel que soit l'état quantique du faisceau lumineux et quelle que soit la fréquence $\Omega$. Si on se restreint au sous-ensemble des "faisceaux classiques " (analogues des états classiques du paragraphe précédent), on obtient alors les limites quantiques standard suivantes pour les variances et les densités spectrales mesurées sur un faisceau lumineux:

$$
\begin{array}{ll}
\Delta I \geq \sqrt{2\langle I\rangle \delta f} & \Delta \varphi \geq \sqrt{\frac{\delta f}{2\langle I\rangle}} \\
S_{l}(\Omega) \geq\langle I\rangle & S_{\varphi}(\Omega) \geq \frac{1}{4\langle I\rangle}
\end{array}
$$

La limite étant atteinte lorsque le faisceau est dans un état cohérent. En particulier, la densité spectrale de bruit dans un faisceau cohérent vaut $S_{I}(\Omega)=\langle I\rangle$. Il s'agit toujours du même bruit de grenaille, ou shot noise, mais généralisé au cas non monomode et analysé spectralement. Ce bruit est indépendant de la fréquence $\Omega$ d'analyse du bruit. On le qualifie de "bruit blanc».

Nous allons voir dans la suite qu'un laser est susceptible d'émettre dans certaines circonstances un faisceau sub-Poissonien, caractérisé par une densité spectrale de bruit d'intensité inférieure à $\langle I\rangle$ dans une bande spectrale $\left[\Omega_{1}, \Omega_{2}\right]$ de largeur déterminée.

\section{BRUIT QUANTIQUE DANS UN AMPLIFICATEUR OPTIQUE}

L'élément constituant de base d'un laser étant un amplificateur optique, il importe de connaître d'abord les caractéristiques du bruit quantique d'un tel système [6]. C'est ce que nous faisons dans cette partie.

\subsection{Relation à la traversée d'un système optique linéaire}

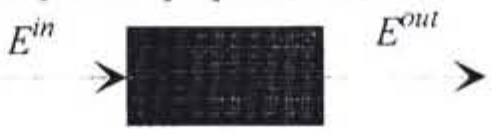

Figure 3

Considérons un système optique (figure 3) qui, lorsqu'il est soumis à un faisceau lumineux entrant monochromatique de fréquence $\omega$, caractérisé par un champ électrique classique $E^{i n}$. produit un faisceau sortant de champ électrique $E^{\text {out }}$ tel que :

$$
E^{\text {out }}=\sqrt{G} E^{\text {in }}
$$

(nous ne traitons pas ici, pour simplifier l'exposé, de l'éventuel déphasage introduit par le système) $\mathrm{Si} G>1$, il s'agit effectivement d'un amplificateur optique, si $G<1$ d'un atténuateur. Quelle est la relation qui va relier les opérateurs quantiques de champ $\hat{E}^{\text {out }}$ et $\hat{E}^{\text {in }}$ mesurés à l'entrée et à la sortie du système optique ? On est tenté de conserver la même relation linéaire et écrire :

$$
\hat{E}^{\text {out }}=\sqrt{G} \hat{E}^{\text {in }}
$$


Il est facile de voir que cette relation implique que les commutateurs de composantes de quadrature entre l'entrée et la sortie du dispositif sont reliés par :

$$
\left[\hat{\mathrm{E}}_{1}^{\text {out }}, \hat{\mathrm{E}}_{2}^{\text {out }}\right]=\mathrm{G}\left[\hat{\mathrm{E}}_{1}^{\text {in }}, \hat{\mathrm{E}}_{2}^{\text {in }}\right]
$$

La relation de commutation (4) devant être valable à la fois pour les champs entrants et sortants, une relation du type (21) n'est acceptable que pour $G=1$, c'est-à-dire dans le cas où le champ n'est ni atténué, ni amplifié dans le processus. Pour assurer la conservation de la relation de commutation dans le cas général, il faut écrire :

$$
\hat{E}^{\text {out }}=\sqrt{G} \hat{E}^{\text {in }}+\hat{B}
$$

L'opérateur $\hat{B}$ rajouté dans l'équation (23) a une valeur moyenne nulle pour que la relation sur les valeurs moyennes quantiques soit la même que la relation (20) entre les valeurs classiques du champ, et satisfait avec son hermitique conjugué à une relation de commutation qui permet à $\hat{E}^{\text {out }}$ d'obéir à la relation de commutation canonique (4). L'opérateur supplémentaire $\hat{B}$ n'intervient donc que dans le calcul des variances. Il traduit le fait que pour $\mathrm{G} \neq 1$, le système optique $n$ 'est pas isolé, mais couple nécessairement les modes entrants et sortants $\hat{E}^{\text {in }}$ et $\hat{E}^{\text {out }}$ à d'autres modes du champ et donc à d'autres sources de bruit. On peut considérer ce phénomène comme un cas particulier du théorème de fluctuation-dissipation : l'atténuation ou l'amplification d'un faisceau lumineux lui rajoute nécessairement des fluctuations venues du réservoir avec lequel le faisceau échange de l'énergie.

\subsection{Cas d'une lame partiellement réfléchissante}

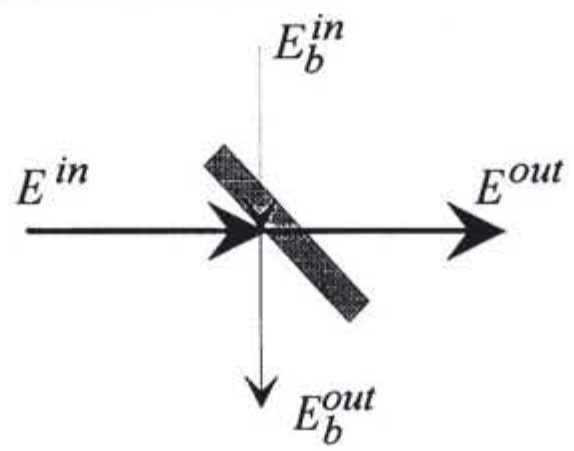

Figure 4

Comme illustration de la relation (23) dans un cas concret simple, nous considérons dans ce paragraphe un attémuateur de faisceau lumineux constitué par une lame partiellement réfléchissante de coefficients de transmission et de réflexion en amplitude $t$ et $r$, que nous supposerons réels et tels que $t^{2}+r^{2}=1$ (figure 4). Il s'agit d'un système à deux entrées et deux sorties, pour lequel on peut écrire la relation suivante entre opérateurs de champ des différents modes :

$$
\hat{E}^{\text {out }}=t \hat{E}^{\text {in }}+r \hat{E}_{b}^{\text {in }}
$$


L'opérateur $\hat{B}$ de la relation (23) est donc dans ce cas particulier égal à $r \hat{E}_{b}^{\text {,n }}:$ il s'agit de la contribution du mode entrant réfléchi sur la lame qui se couple à la voie de sortie. Quant à $G$,il vaut $t^{2}$. Il est facile alors de montrer qu'une telle relation assure la conservation des relations de commutation :

$$
\left[\hat{E}_{1}^{\text {out }}, \hat{E}_{2}^{\text {out }}\right]=t^{2}\left[\hat{E}_{1}^{\text {in }}, \hat{E}_{2}^{i n}\right]+r^{2}\left[\hat{E}_{b 1}{ }^{i n}, \hat{E}_{b 2}{ }^{i n}\right]=\left(t^{2}+r^{2}\right) 2 i E_{\omega}^{2}=2 i E_{\omega}^{2}
$$

L'état du champ entrant par cette voie est le vide. En conséquence, les relations pour le champ moyen s'écrivent :

$$
\left\langle\hat{E}^{\text {out }}\right\rangle=t\left\langle\hat{E}^{i n}\right\rangle
$$

et pour les variances des composantes de quadrature $\mathrm{E}_{\mathrm{i}}(\mathrm{i}=1,2)$ :

$$
\left(\Delta E_{i}^{\text {our }}\right)^{2}=t^{2}\left(\Delta E_{i}^{\text {in }}\right)^{2}+r^{2} E_{\omega}^{2}
$$

On voit bien que les fluctuations du vide se rajoutent sur la voie de sortie à cause de la présence de cette deuxième voie d'entrée du système.

\subsection{Cas de l'émission stimulée}

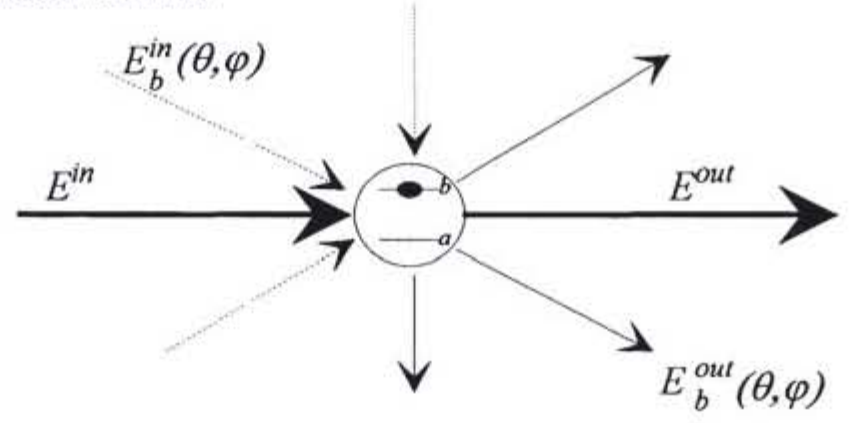

Figure 5

L'amplification laser est basée sur le phénomène d'émission stimulée sur une transition $a \rightarrow b$ présentant une inversion de population (figure 5). L'émission stimulée est toujours accompagnée du phénomène de diffusion résonnante qui couple par émission spontanée le

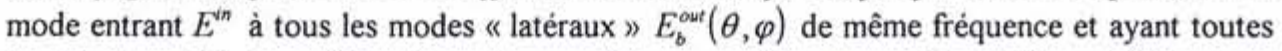
les directions $(\theta, \varphi)$ possibles. Le milieu amplificateur «fluoresce » c'est-à-dire qu'il émet une partie de sa lumière dans les directions autres que celle du faisceau incident, avec une certaine amplitude de probabilité $s(\theta, \varphi, \omega)$. On peut alors faire un raisonnement analogue à celui fait dans le cas de la lame réfléchissante : le même processus de diffusion couple les voies entrantes inoccupées $E_{b}^{\text {in }}(\theta, \varphi)$ au mode sortant $E^{\text {out }}$, avec - au signe près - la même amplitude de probabilité. L'opérateur de bruit $\hat{B}$ de la relation (23) provient de la contribution de tous ces modes latéraux. En conséquence les fluctuations du vide entrant par les modes latéraux vont, par ce processus de diffusion résonnante, ajouter du bruit sur le mode sortant. Ce terme dû à l'émission spontanée n'est que le bruit minimum rajouté à l'amplification. Le mécanisme d'inversion lui-même, responsable de l'existence du gain, peut présenter des fluctuations («bruit de pompage ») qui se rajouteront à ce bruit minimum. 


\subsection{Excès de bruit dans un amplificateur linéaire}

Un raisonnement général à partir de la relation (23) et donc indépendant du mécanisme d'amplification précis [6] montre que les variances des composants de quadrature du champ amplifié sont données, au mieux, par :

$$
\left(\Delta E_{i}^{\text {out }}\right)^{2}=G\left(\Delta E_{i}^{\text {in }}\right)^{2}+(G-1) E_{\omega}^{2}
$$

$G$ étant est le coefficient d'amplification en énergie de l'amplificateur optique. La relation (28) montre que si l'état entrant est cohérent $\left(\left(\Delta E_{i}^{i n}\right)^{2}=E_{\omega}^{2} \quad i=1,2\right)$, l'état sortant a un bruit égal à (2G-1) fois le bruit entrant. On a donc un facteur de bruit différent de 1 . Ce facteur $\mathrm{F}$ est, rappelons le, égal au gain du système relatif aux bruits entrant et sortant divisé par le gain du système relatif aux signaux entrant et sortant. Dans le cas présent :

$$
\mathrm{F}=\frac{2 G-1}{G}=2-\frac{1}{G}
$$

Pour de grands facteurs d'amplification (et rappelons le pour un mode entrant cohérent), F vaut 2 : ce sont les fameux « $3 \mathrm{~dB} »$ de bruit introduits par l'amplification optique.

\subsection{Amplificateur dépendant de la phase}

Il existe d'autres systèmes optiques, dans lesquels le gain dépend de la phase du champ incident. Il faut donc écrire des relations différentes pour les deux composantes de quadrature du champ classique :

$$
E_{1}^{\text {out }}=\sqrt{G_{1}} E_{1}^{\text {in }} \quad E_{2}^{\text {out }}=\sqrt{G_{2}} E_{2}^{\text {in }} \quad G_{1} \neq G_{2}
$$

Les relations (30) ne peuvent se transposer aux opérateurs quantiques tout en assurant la conservation des relations de commutation entre l'entrée et la sortie que dans le cas particulier où :

$$
\mathrm{G}_{1} \mathrm{G}_{2}=1
$$

On a alors :

$$
\hat{E}_{1}^{\text {out }}=\sqrt{G_{1}} \hat{E}_{1}^{\text {in }} \quad \hat{E}_{2}^{\text {out }}=\frac{1}{\sqrt{G_{1}}} \hat{E}_{2}^{\text {in }}
$$

Une telle transformation ne comporte pas de terme d'excès de bruit, à la différence de l'expression (23). Supposons $G_{1}>1$ par exemple : la composante de quadrature $E_{1}$ est donc amplifiée sans aucun bruit rajouté (le facteur de bruit vaut 1). En revanche, la composante de quadrature $E_{2}$ est, elle, atténuée (mais aussi sans bruit ajouté, à la différence de la lame partiellement réfléchissante). Un tel dispositif optique n'est donc amplificateur que pour une phase bien déterminée du champ entrant. On montre qu'un amplificateur paramétrique dégénéré (modes signal et idler de même vecteur d'onde et de même polarisation) obéit à des relations du type (32), la phase sur laquelle le champ est amplifié étant reliée à la phase du champ pompe. Notons en outre que si on met un état cohérent à l'entrée de ce type d'amplificateur, on obtient à la sortie un état comprimé.

\subsection{Amplificateur avec saturation du gain}

On a vu que dans un laser fonctionnant en régime stationnaire, l'amplificateur optique 
fonctionne en régime saturé, dans lequel le gain dépend de l'intensité du champ entrant. On a donc la relation classique suivante :

$$
E^{\text {out }}=\sqrt{G\left(\left|E^{\text {in }}\right|^{2}\right)} E^{\text {in }}
$$

Calculons le gain d'un tel amplificateur pour de petites variations $\delta E^{\text {in }}$ autour d'un champ entrant moyen $\bar{E}^{i n}$ («gain petit signal »). Nous supposerons la phase du champ entrant nulle pour simplifier. En supposant $\delta E^{\text {in }}$ petit devant $\bar{E}^{\text {in }}$, il est possible de linéariser les équations donnant le signal de sortie $\delta E^{\text {out }}$ autour de la valeur moyenne $\bar{E}^{\text {out }}$. On obtient alors les équations suivantes pour les deux composantes de quadrature de $\delta E^{\text {out }}$ :

$$
\left\{\begin{array}{c}
\delta E_{1}^{\text {out }}=\frac{\bar{G}+\left(\bar{E}^{\text {in }}\right)^{2} \bar{G}^{\prime}}{\sqrt{\bar{G}}} \delta E_{1}^{\text {in }} \\
\delta E_{2}^{\text {out }}=\sqrt{\bar{G}} \delta E_{2}^{\text {in }}
\end{array}\right.
$$

où $\bar{G}=G\left(\left(\bar{E}^{i n}\right)^{2}\right)$ et $\bar{G}^{\prime}=\frac{d G}{d\left(E^{i n}\right)^{2}}$. Ces relations montrent bien que le gain $d^{\prime} u n$ amplificateur saturé dépend de la phase. Il est différent pour la composante de quadrature $\delta E_{2}$ perpendiculaire au champ moyen, qui décrit les fluctuations de phase du champ entrant et dont la variation n'affecte pas - au premier ordre - l'intensité du champ, donc la valeur du gain, et pour la composante $\delta E_{1}$, qui décrit les fluctuations d'amplitude du champ entrant. Les propriétés de bruit de ce type d'amplificateur ne sont donc pas celles décrites dans le paragraphe 2.4. (il n'y a pas nécessairement $3 \mathrm{~dB}$ de bruit rajouté). Il se peut en particulier que cet amplificateur ne rajoute aucun bruit lorsque le produit des deux gains vaut 1 , c'est-à-dire lorsque $\overline{\mathrm{G}}+\left(\overline{\mathrm{E}}^{\text {in }}\right)^{2} \mathrm{G}^{\prime}=1$. Il est facile de voir que dans ce cas, $G$ est inversement proportionnel à $\left|E^{i n}\right|^{2}$ : on est alors dans le régime de saturation complète, oủ l'intensité de sortie $G\left|E^{i n}\right|^{2}$ ne dépend plus de l'intensité entrante. Un tel système atténue les fluctuations entrantes d'intensité, et amplifie les fluctuations de phase, sans rajouter de bruit.

\section{BRUIT QUANTIQUE DANS UN LASER}

\subsection{Généralités}

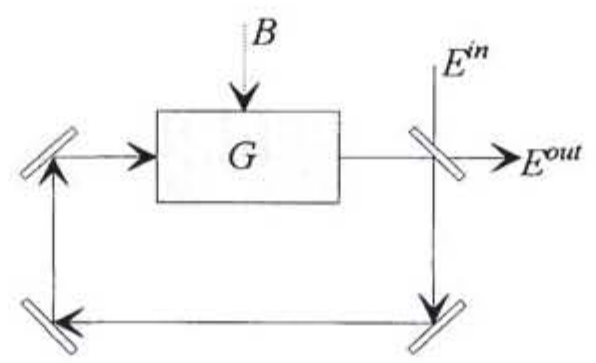

Figure 6 
La figure 6 donne le schéma simplifié d'un laser, montrant les différentes origines possibles du bruit qui va affecter le champ de sortie $E^{\text {out }}$ de ce laser :

- le mode $E^{i n}$ couplé au mode intracavité du champ laser, qui va introduire dans la cavité les fluctuations du vide de ce mode (si on prend un laser non injecté).

- l'excès de bruit de l'amplificateur $B$, c'est-à-dire l'émission spontanée inséparable de l'émission stimulée à l'origine de l'effet laser.

- les fluctuations du gain $G$, liées à la dynamique du processus qui crée l'inversion de population, et que nous appellerons bruit de pompage.

Notons d'emblée que les modes d'entrée et de sortie de l'amplificateur laser ne sont pas des champs libres, puisqu'ils sont en fait le même mode d'une cavité optique, et que l'amplificateur optique fonctionne en régime saturé : on ne peut donc pas appliquer à cet amplificateur les limites quantiques obtenues pour l'amplificateur linéaire isolé de la partie 2.1. Les limites quantiques porteront uniquement sur la relation globale d'entrée-sortie qui relie les fluctuations $\delta E^{\text {out }}$ du champ de sortie aux fluctuations $\delta E^{\text {in }}$ du champ entrant (qui peuvent être les fluctuations du vide).

On sait que lorsque le laser fonctionne au-dessus du seuil, son intensité stationnaire est fixée par l'égalité gain saturé = pertes, mais que sa phase est quelconque. Les fluctuations auxquelles le laser est soumis $\left(\delta \mathrm{E}^{\text {in }}, \delta \mathrm{B}, \delta \mathrm{G}\right)$ vont avoir tendance à « déstabiliser » le laser en l'écartant de son point de fonctionnement initial. Celui-ci réagit en ramenant l'intensité à sa valeur moyenne avec une certaine constante de temps, par définition même de la stabilité du fonctionnement du laser. Le bruit d'intensité de sortie du laser dépendra des caractéristiques dynamiques de cette «force de rappel » sur l'intensité du champ. En revanche, comme la phase du champ n'a pas une valeur déterminée en régime stationnaire, le laser ne réagira pas aux variations de phase induites par les bruits entrants. La phase évoluera donc librement, sans «force de rappel », et ses variations ne seront donc pas bornées : il y aura diffusion de phase dans le laser. Comme dans tout processus de diffusion, la variance de la phase $(\Delta \varphi)^{2}$ croît proportionnellement au temps. Elle est infinie pour des temps très longs. En d'autres termes, la densité spectrale du bruit de phase diverge pour une fréquence de bruit mulle :

$$
t \rightarrow \infty \quad \Delta \varphi \rightarrow \infty \text { ou } S_{\varphi}(\Omega=0) \rightarrow \infty
$$

Cette propriété est intrinsèque à tout laser, dans lequel il n'existe aucune référence de phase sur laquelle le champ sortant puisse s'accrocher (cela n'est évidemment pas vrai pour le laser injecté). On sait qu'il existe des relations d'Heisenberg (16) ou (18) entre bruit d'intensité et bruit de phase. La relation (35) implique qu'il n'y a pas de limite inférieure imposée par la mécanique quantique à $\Delta I$, ou bien à la densité spectrale du bruit d'intensité à fréquence mulle $S_{l}(\Omega=0)$. La mécanique quantique n'interdit donc pas l'existence de lasers ayant un bruit d'intensité nul à basse fréquence ("lasers sub-Poissoniens»), à cause précisément du phénomène de diffusion de phase. C'est la nature et l'importance des différentes sources de bruit qui vont déterminer si un laser donné peut fonctionner dans un tel régime sub-Poissonien.

\subsection{Modèle simple}

Pour évaluer l'importance des différentes sources de bruit, considérons un laser pour lequel existe la relation suivante entre champ entrant $E$ et $E^{\prime}$ sortant de l'amplificateur optique :

$$
E^{\prime}=(1+g) E+\bar{E} \delta g+\delta B
$$


$g$ est un terme de gain optique, supposé réel et petit devant 1 qui dépend de l'intensité intracavité sous la forme (voir cours sur les principes de fonctionnement des lasers $§ 2.4 .2$ ) :

$$
\mathrm{g}=\frac{\mathrm{g}_{0}}{1+\mathrm{I} / \mathrm{I}_{\mathrm{s}}} \quad \mathrm{g}_{0}: \text { gain non saturé }
$$

On suppose de plus la cavité laser sans pertes internes et de grande finesse (la transmission en intensité $T$ du miroir de couplage est petite devant 1 ). Le régime stationnaire d'un tel système est déterminé par l'équation d'égalité du gain et des pertes soit : (cours sur les principes du laser $\S 1.2$ )

$$
g(\bar{I})=\frac{T}{2}=\frac{\kappa L}{c}
$$

où $L$ est la longueur optique de la cavité laser. Les fluctuations $\delta E$ autour de cette valeur moyenne obéissent à l'équation d'évolution du laser (cours sur les principes du laser $\$ 4.1$, équation (58) dans le cas $g L_{\mathrm{A}} \ll<1, A=T<<1$ ) linéarisée autour de la valeur $\bar{E}$ donnée par le régime stationnaire :

$$
\frac{\mathrm{L}}{\mathrm{c}} \frac{\mathrm{d}}{\mathrm{dt}}(\delta \mathrm{E})=\left[\mathrm{g}(\mathrm{I})-\frac{\kappa \mathrm{L}}{\mathrm{c}}\right] \delta \mathrm{E}+\overline{\mathrm{E}} \delta \mathrm{g}+\sqrt{\mathrm{T}} \delta \mathrm{E}^{\mathrm{in}}+\delta \mathrm{B}
$$

Par un calcul analogue à [7], cette équation permet de déterminer la valeur des composantes de Fourier des fluctuations $\delta E$ du champ intracavité, puis celles du champ sortant du laser. Si on connaît les propriétés statistiques de $\delta B$ et $\delta g$, on peut ainsi calculer la densité spectrale des bruits d'intensité et de phase du champ laser dans le cadre de ce modèle simple. Nous n'indiquons ici que les résultats d'un tel calcul.

\subsubsection{Bruit de phase}

On montre que la variance des fluctuations de phase du champ intracavité s'écrit dans ce modèle :

$$
\left\langle|\varphi(t)-\varphi(0)|^{2}\right\rangle=2 D_{S T} t
$$

où $D_{S T}$ est un coefficient de diffusion de la phase, appelé coefficient de diffusion SchawlowTownes [8], et qui vaut simplement :

$$
D_{S T}=\frac{\kappa}{\bar{N}}
$$

oú $\bar{N}=\bar{I} \frac{L}{c}$ est le nombre moyen de photons présent dans le mode laser intracavité. Le calcul montre que $D_{S T}$ a une double origine : les fluctuations du vide $E^{\text {in }}$ entrant par le miroir de couplage et le bruit $\delta B$ d'émission spontanée. Le bruit de pompage n'influe en revanche pas sur la diffusion de phase. On peut aussi calculer la densité spectrale du bruit de phase $S_{\varphi}(\Omega)$, puis celle du bruit de fréquence $S_{\omega}(\Omega)\left(S_{\omega}(\Omega)\right.$ est égal à $\Omega^{2} S_{\varphi}(\Omega)$, car la fréquence instantanée $\omega$ n'est autre que la dérivée temporelle de la phase $\varphi$ ) :

$$
S_{\varphi}(\Omega)=\frac{D_{S T}}{\Omega^{2}} \quad S_{\omega}=D_{S T}
$$


On a donc bien une divergence à fréquence nulle du bruit de phase, comme prévu dans le paragraphe précédent (Figure 7a).

On peut enfin déterminer la répartition spectrale d'intensité du champ laser $I(\omega)$ (mesurée en utilisant un spectromètre optique à prisme ou à réseau). Elle vaut dans ce modèle :

$$
I(\omega)=\frac{\bar{I}}{\pi} \frac{D_{S T}}{\left(\omega-\omega_{0}\right)^{2}+D_{S T}^{2}}
$$

Le champ laser intracavité a donc une forme de raie Lorentzienne de largeur $2 D_{S T}$. Il s'agit évidemment du cas d'un "laser théorique », où seuls les bruits quantiques entrent en jeu dans la largeur de raie. Dans les lasers réalisés en pratique, d'autres sources de bruit influent sur cette largeur (voir cours de C. Chardonnet).

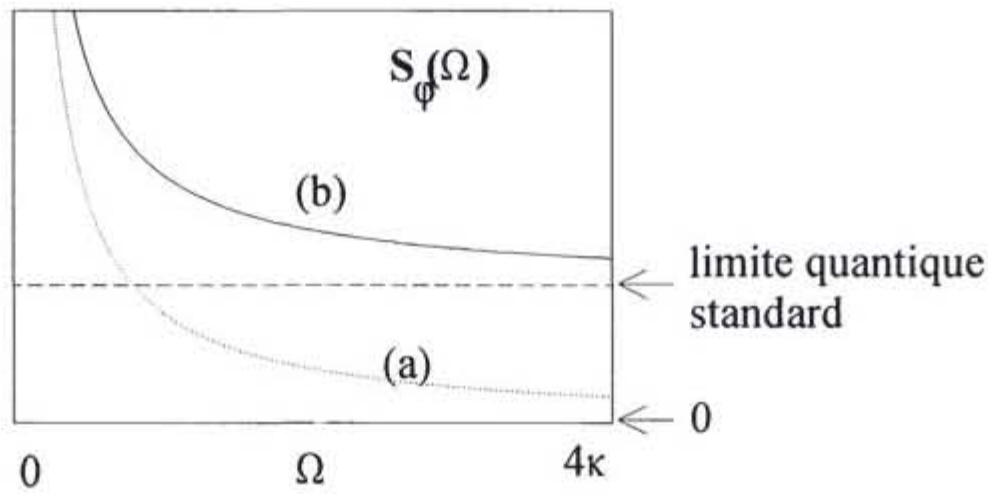

Figure 7

Si on calcule maintenant les fluctuations de phase du champ sortant du laser, on obtient la densité spectrale suivante pour le bruit de phase (figure $7 \mathrm{~b}$ ) :

$$
S_{\Phi}^{\text {out }}=\frac{D_{S T}}{\Omega^{2}}+\frac{1}{4 I^{\text {out }}}
$$

On retrouve à basse fréquence les expressions (42) du champ intracavité. A haute fréquence, c'est-à-dire pour des fréquences de bruit plus grandes que la bande passante $\kappa$ de la cavité laser, le bruit de phase tend en revanche vers une constante qui n'est autre que la limite quantique standard du bruit de phase (équation 19).

Le coefficient Schawlow-Townes joue ainsi un grand rôle dans les problèmes de bruit de phase d'un laser. Donnons-en quelques expressions équivalentes:

$$
D_{S T}=\frac{\kappa}{\bar{N}}=\frac{\kappa \hbar \omega_{0}}{T_{\text {cov }} \mathrm{P}}=\frac{2 \kappa^{2} \hbar \omega_{0}}{\mathrm{P}_{\text {out }}}
$$

oủ $\mathrm{P}$ et $\mathrm{P}_{\text {out }}$ sont respectivement les puissances du champ laser (en W) intracavité et sortantes. La largeur Schawlow-Townes $D_{S T} / 2 \pi$ est typiquement de l'ordre du $\mathrm{mHz}$ pour un laser Hélium Néon $\left(\mathrm{P}_{\text {out }}=1 \mathrm{~mW}, \lambda=0,6 \mu \mathrm{m}, \mathrm{T}=2 \% \mathrm{~L}=2 \mathrm{~m}\right)$ et du $\mathrm{MHz}$ pour un laser à semi-conducteur $\left(\mathrm{P}_{\text {out }}=1 \mathrm{~mW}, \lambda=1 \mu \mathrm{m}, \mathrm{T}=10 \% \mathrm{~L}=100 \mu \mathrm{m}\right)$. 


\subsubsection{Bruit d'intensité}

A partir de (39) et de la connaissance des caractéristiques des bruits d'émission spontanée et de pompage, il est possible de calculer le bruit d'intensité du champ sortant. On peut en particulier utiliser un modèle de type coefficient d'Einstein pour la dynamique du système à 2 niveaux (cours sur les principes des lasers $\S 2.3$ ) et tenir compte des fluctuations $\delta \Lambda_{b}$ du taux de pompage $\Lambda_{b}$ du niveau supérieur de la transition en prenant une fonction de corrélation de cette quantité de la forme $[9,10]$ :

$$
\left\langle\delta \Lambda_{b}(t) \delta \Lambda_{b}\left(t^{\prime}\right)\right\rangle=\langle\Lambda\rangle C_{p} \delta\left(t-t^{\prime}\right)
$$

$C_{P}$ caractérise le bruit de pompage : il est nul pour un pompage régulier, sans fluctuations, et égal à 1 pour un pompage "Poissonien», qui est un cas souvent rencontré (pompage optique avec champ cohérent, variations statistiquement indépendantes des différents éléments actifs dans l'amplificateur...). (Des calculs analogues à celui présenté succinctement ici figurent dans les références [9-11]). On obtient l'expression suivante pour le bruit d'intensité du faisceau de sortie du laser :

$$
S_{I}^{\text {out }}(\Omega)=\bar{I}^{\text {out }}\left[1+\frac{\kappa^{2}}{\Omega^{2}+\kappa^{2}(1-1 / R)^{2}}\left\{\frac{2}{R}+\left(1-\frac{1}{R}\right) \frac{\gamma_{b}}{\gamma_{a}+\gamma_{b}}\left(C_{P}-1\right)\right\}\right]
$$

oủ $\gamma_{a}$ et $\gamma_{b}$ sont les taux de désexcitation des niveaux inférieur et supérieur de la transition laser (pour la plupart des lasers $\gamma_{a}<<\gamma_{b}$, $) \times$ le taux de décroissance du champ dans la cavité vide, et $R=\Lambda_{b} /\left(\Lambda_{b}\right)_{\text {seuti }}$ le taux de pompage, égal à 1 au seuil d'oscillation du laser.

La figure (8) donne quelques exemples de spectres de bruit d'intensité dans le cas $\gamma_{a}<<\gamma_{b}$ :-

- Dans la figure $8 \mathrm{a}, \mathrm{R}$ vaut 1,5 ( $50 \%$ au dessus du seuil), et les différentes courbes correspondent aux valeurs de CP égales à $0 \quad 0,25 \quad 0,5 \quad 0,75$ et 1 . On voit que, lorsqu'on est près du seuil, le bruit d'intensité à basse fréquence est largement au dessus de la limite quantique standard. Il est d'autant plus important qu'on est plus proche du seuil, c'est-à-dire que la proportion de photons émis spontanément dans le mode laser sur le nombre de photons " stimulés » est plus grand. Il ne dépend que très peu du bruit de pompage, parce qu'il est dominé par le bruit d'émission spontanée.

- Dans la figure $8 \mathrm{~b}, R$ est très grand $(R=20)$, et les différentes courbes en partant de la plus basse correspondent aux valeurs de $C_{P}$ égales à $00,250,50,75$ et 1 . On constate que, lorsqu'on est très au dessus du seuil, le bruit d'intensité basse fréquence est dominé par le bruit de pompage. La formule (47) montre qu'il tend vers $C_{P} I^{\text {put }}$ à taux de pompage $R$ infini. Il y a en fait strict transfert de la statistique de pompage à la statistique des photons sortant du laser. Le laser est dès lors sub-Poissonien lorsque la statistique de pompage est subPoissonienne. Ce comportement a été prédit pour la première fois par Golubev et Sokolov [12] puis par Yamamoto [11].

- On constate enfin que dans tous les cas, pour les fréquences de bruit supérieures à la bande passante de la cavité laser $S_{I}^{\text {out }}(\Omega)$ tend vers $I^{\text {out }}$ c'est-à-dire vers la limite quantique standard pour le bruit d'intensité (shot noise). 


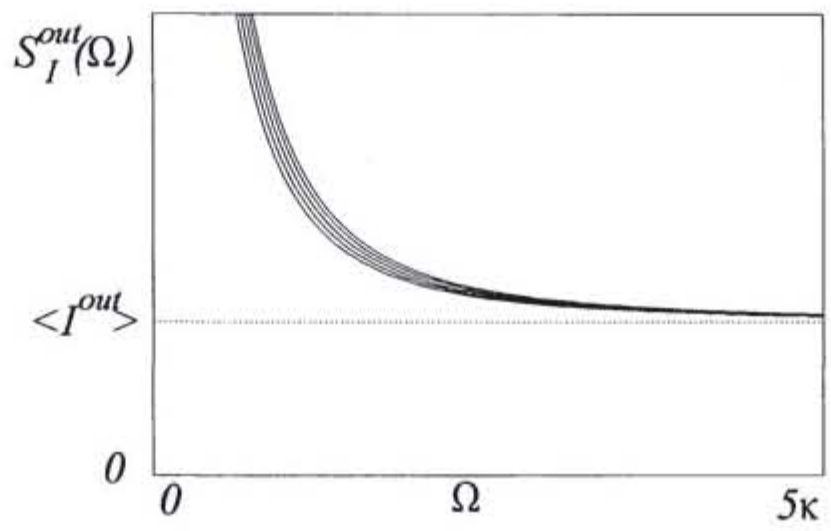

a)

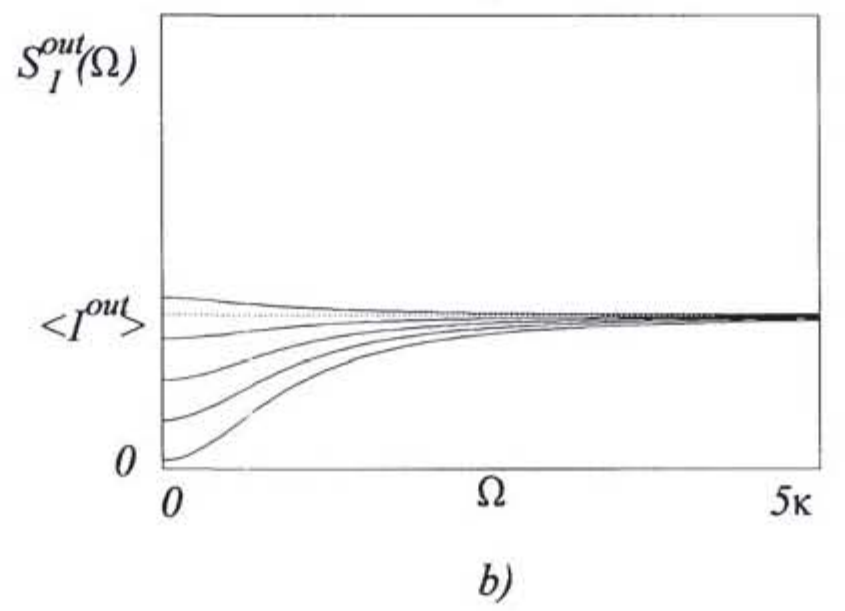

Figure 8

\subsection{Cas où le gain a une partie imaginaire non négligeable}

Nous avons vu dans le cours sur les principes des lasers $(\S 2)$ que pour un système à deux niveaux le gain $g$ était de la forme :

$$
g=\frac{\chi_{e}^{\prime}+i \chi_{e}^{\prime \prime}}{1+I / I_{S}}\left(k L_{A} / 2\right)
$$

La partie imaginaire de $g$, qui est reliée à l'indice du milieu actif, dépend aussi de l'intensité lumineuse intracavité, donc de ses fluctuations. En conséquence, les fluctuations de phase du champ laser, qui sont liées aux fluctuations de la longueur optique de la cavité sont dans ce cas couplées aux fluctuations d'intensité du champ intracavité. On peut écrire les fluctuations de gain $\delta \mathrm{g}$ sous la forme : 


$$
\delta g=\delta g_{R}(1-i \alpha) \text { avec } \alpha=-\frac{\left(\partial \chi^{\prime \prime} / \partial I\right)}{\left(\partial \chi^{\prime} / \partial I\right)}
$$

Dans le modèle simple du $\S 3.2$. du cours sur les principes des lasers, $\alpha$ vaut $\frac{\omega_{0}-\omega}{\gamma_{a b}}$ ou encore $\frac{\omega_{0}-\omega_{m}}{\gamma_{a b}+\kappa}$ (où $\omega_{m}$ est la fréquence propre de la cavité laser). En utilisant l'expression (49), on peut montrer que le coefficient de diffusion a une valeur $D$ modifiée par rapport au modèle simple précédent. On trouve [13] :

$$
D=D_{S T}\left(1+\alpha^{2}\right)
$$

$\alpha$, négligeable dans les lasers à gaz où les milieux amplificateurs sont dilués, prend des valeurs importantes (typiquement entre 3 et 6 ) pour les lasers à semi-conducteurs.

\subsection{Modèle cinétique complet}

Dans le modèle simple exposé dans la partie 3.2., le gain était supposé avoir une valeur déterminée uniquement par la valeur instantanée du champ ittracavité : il supposait donc une réponse instantanée des atomes aux variations du champ, ce qui n'est pas le cas de tous les lasers. Il est possible de faire une théorie complète, tenant compte de la dynamique couplée des atomes et de la cavité, en écrivant des équations cinétiques pour le champ intracavité $E$, les populations des niveaux a et b de la transition laser, et la cohérence $\sigma_{\alpha \beta}$ entre ces deux niveaux (voir cours sur les principes des lasers \$2.1), en incluant des termes de bruit liés aux différents processus de relaxation et de pompage. Un tel calcul est par exemple effectué dans la référence [9] pour un modèle particulier de pompage. Nous donnons ici les résultats importants auxquels aboutit ce type d'approche.

En ce qui concerne le bruit de phase, on obtient ici aussi un processus de diffusion de phase, avec une constante de diffusion $D$ qui vaut :

$$
D=D_{s T}\left(\frac{\gamma_{a b}}{\gamma_{a b}+\kappa}\right)^{2}
$$

où $\gamma_{\mathrm{ab}}$ est le taux de relaxation du dipôle atomique, et $\kappa=T \frac{c}{L}$ celui de l'amplitude du champ intracavité. Dans le cas où la relaxation du dipôle atomique est très rapide devant celle du champ $\left(\gamma_{\mathrm{ab}}>>_{\mathrm{K}}\right)$, on retrouve bien le résultat du modèle précédent. Dans le cas inverse, la diffusion Schawlow-Townes est ralentie par un facteur $\left(\gamma_{a b} / \kappa\right)^{2}$, car le dipôle atomique n'arrive pas à suivre les fluctuations du champ $[14,15]$. Notons enfin que le bruit de phase ne dépend pas des caractéristiques du bruit de pompage.

En ce qui concerne le bruit d'intensité, on retrouve les conclusions du paragraphe 3.2. dans le cas où les atomes sont " rapides » par rapport à la cavité. Dans le cas inverse, on obtient des spectres de bruit d'intensité ayant l'allure de la figure 9 , donnée à la limite de très fort pompage : $R=10^{4}$, et pour $\gamma_{a b}=10^{3} \kappa, \gamma_{a}=10^{-3} \kappa, \gamma_{b}=0.1 \kappa$. Les différentes courbes, en partant de la plus basse, correspondent aux valeurs de $C_{P}$ égales à $00,250,50,75$ et 1 .

On observe un excès de bruit vers $\Omega=5 \mathrm{~K}$, dû à la présence d'oscillations de relaxation dans le laser (comme toujours, le bruit d'intensité tend vers le shot noise pour des fréquences de bruit encore plus élevées). 


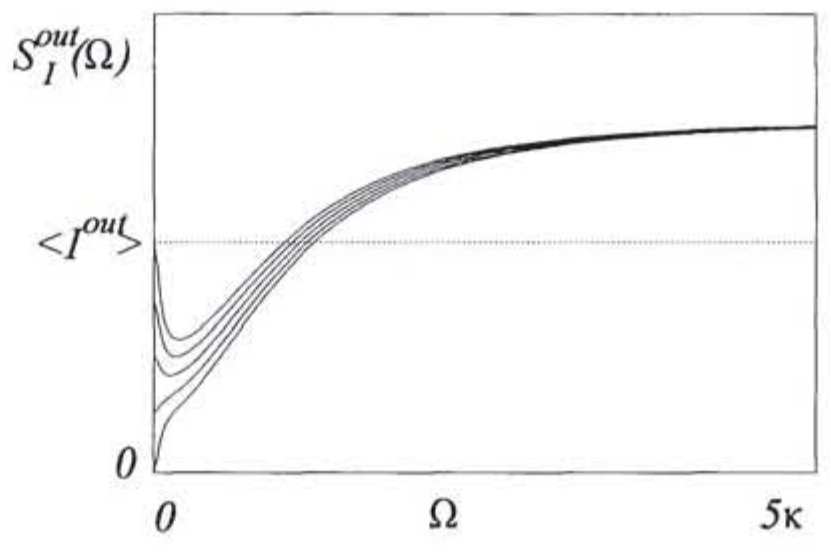

Figure 9

Le bruit à fréquence nulle vaut toujours $C_{P}$ fois le shot noise, et s'annule donc en cas de pompage régulier. Le fait nouveau est que pour les fréquences intermédiaires, on peut obtenir un rayonnement sub-Poissonien même dans le cas d'un pompage Poissonien $\left(C_{p}=1\right.$ courbe supérieure de la figure 9). La valeur maximale de réduction de bruit est de $50 \%$ : c'est ici la dynamique de la réponse atomique au pompage qui régularise le flux des photons laser.

Ce type de comportement a été trouvé dans le cadre d'autres modèles théoriques pour le processus de pompage (pompage sur un cycle fermé de transitions [16], avec un maximum de réduction de $50 \%$ ) ou bien pour les processus de relaxation (relaxation collective dans le laser "superradiant» [17] avec une réduction de bruit approchant de $100 \%$ dans certaines configurations).

\subsection{Un laser émet-il un état cohérent ?}

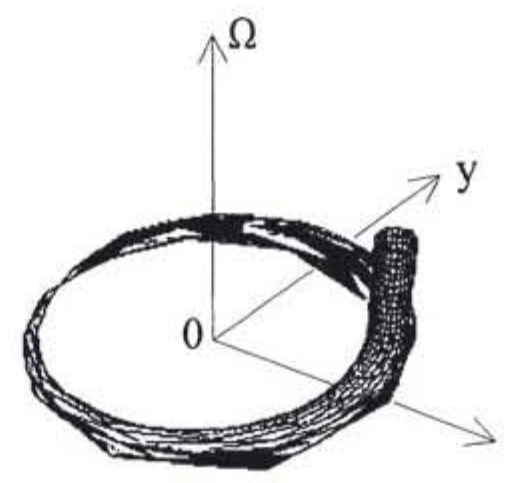

Figure 10 
Nous avons passé en revue l'effet sur le champ laser des différentes sources de bruit su sur la figure tridimensionnelle (10), qui donne, comme les figures (1) et (2), la surface d'incertitude pour l'extrémité du vecteur de Fresnel, mais dans un plan xOy horizontal, la coordonnée verticale étant la fréquence de bruit $\Omega$ autour de laquelle on analyse le bruit.

- Pour les fréquences de bruit petites devant la bande passante de la cavité $(\Omega<<\kappa)$, la surface de bruit prend une forme annulaire : le bruit de phase, dû à la diffusion Schawlow-Townes est très grand, alors que le bruit d'intensité est gouverné par le bruit de pompage et la dynamique du couplage champ-atomes-pompage. En particulier, la largeur de l'anneau peut être inférieure à $E_{\omega}$ (laser sub-Poissonien).

- Pour $\Omega \gg \kappa$, la surface d'incertitude tend vers un cercle, correspondant au bruit d'un état cohérent et le bruit du laser, à la fois pour la phase et l'intensité, tend vers la limite quantique standard.

Un laser n'émet donc de manière certaine un état cohérent que lorsqu'on s'intéresse à des phénomènes rapides devant la durée de vie de la cavité.

\section{LASERS SUB-POISSONIENS À POMPAGE RÉGULIER}

Nous avons vu qu'à basse fréquence de bruit, lorsque le laser fonctionne très au dessus du seuil, et si le milieu amplificateur évolue naturellement plus rapidement que le champ dans la cavité, le bruit d'intensité du laser n'est autre que le bruit du processus de pompage. Pour obtenir alors un laser à bruit d'intensité comprimé, il faut donc utiliser un pompage régulier. Différents schémas de pompage régulier ont été envisagés.

\subsection{Pompage par impulsions}

Golubev et Sokolov [12] ont montré qu'on obtenait un pompage régulier avec un laser comportant un nombre fixe $N$ d'atomes actifs, pompés optiquement par des impulsions régulières. L'intensité de celles-ci est déterminée de telle sorte qu'à la fin de chaque impulsion les $N$ atomes se trouvent tous dans l'état excité b, l'intervalle entre impulsions étant suffisamment long pour que les $N$ atomes se retrouvent dans l'état fondamental juste avant l'impulsion suivante ; un nombre fixe $N$ de photons est donc déposé dans la cavité à chaque cycle, ce qui conduit à un bruit d'intensité nul à fréquence nulle. Ce schéma séduisant n'a cependant jamais été réalisé expérimentalement jusqu'à présent.

\subsection{Laser à semi-conducteur pompé par courant électrique}

L'idée théorique, et la réalisation expérimentale d'un laser à semi-conducteur sub-Poissonien, reviennent à Yamamoto et à son équipe [11]. L'idée de base est la suivante : dans un laser à semi-conducteur dont le rendement est de $100 \%$, toute annihilation électron-trou au niveau de la jonction produit un photon. La statistique de passage des électrons est donc transférée sans altérations sur la statistique d'émission des photons, ou, en d'autres termes, le bruit de pompage n'est autre que bruit du courant électrique d'injection $i$. Or dans un circuit électrique, le bruit du courant $i$ n'est lié qu'au processus dissipatif qui se produit dans la résistance $R$ du circuit, c'est-à-dire au bruit thermique de la résistance (bruit Nyquist), dont la densité spectrale vaut :

$$
S_{i}(\Omega)=\frac{2 k_{B} T}{R}
$$


(Les électrons dans le circuit sont en effet en régime diffusif et non balistique : il n'y a donc pas de bruit de grenaille associé au passage d'un courant électrique); Lorsque le laser fonctionne très au dessus du seuil, c'est ce bruit que l'on va observer sur l'intensité du faisceau de sortie à fréquence de bruit nulle. Chaque charge $q$ donnant un photon, la densité spectrale du flux numérique $I$ de photons sortant vaut alors :

$$
S_{I}(\Omega=0)=\frac{2 k_{B} T}{q^{2} R}
$$

Cette valeur est à comparer au bruit de grenaille, dont la densité spectrale est égale au flux numérique moyen de photons $\bar{I}=\frac{\bar{i}}{q}$ ( $\bar{i}$ : courant électrique moyen dans le circuit d'injection). Le faisceau de sortie sera donc sub-Poissonien, ou bien en dessous de la limite quantique standard, dès lors que :

$$
\frac{2 k_{B} T}{q^{2} R}<\frac{\bar{i}}{q} \Leftrightarrow R \bar{i}>\frac{2 k_{B} T}{q} \text { (=52mV à la température ambiante) }
$$

Pour obtenir un laser à semi-conducteur sub-Poissonien, il suffit donc de mettre dans le circuit d'alimentation de la diode une résistance suffisamment forte pour que l'inégalité (54) soit satisfaite.

Expérimentalement, les choses sont évidemment plus compliquées, parce qu'il est difficile d'obtenir à la fois un rendement quantique proche de 1 , un fonctionnement strictement monomode très au dessus du seuil et pas d'autres sources de bruit que le bruit de pompage (le bruit d'un laser à semi-conducteur est en particulier très sensible à toute rétrodiffusion de lumière). Ces conditions ont été réalisées expérimentalement [18] pour un laser refroidi à la température de l'Hélium liquide et en utilisant un détecteur situé lui aussi dans le cryostat. Le résultat est impressionnant puisqu'une réduction de bruit de $85 \%$ sur une large bande de fréquence de bruit a été obtenue.

On s'est récemment rendu compte qu'on pouvait obtenir des réductions de bruit non négligeables avec des diodes lasers du commerce et, à la température ambiante, si on forçait le fonctionnement monomode de la diode laser en utilisant une cavité extérieure comprenant un réseau optique pour sélectionner une fréquence, ou bien en utilisant un laser d'injection [19]. Une expérience « de coin de table » permet donc maintenant d'obtenir un laser sub-Poissonien dont le bruit est réduit d'environ $20 \%$ par rapport au shot noise. C'est un exemple frappant des progrès extrêmement rapides à la fois sur la plan théorique et expérimental, qui ont été accomplis dans le domaine de l'étude du bruit quantique des faisceaux lumineux au cours des toutes dernières années.

\section{Références}

[1] Reynaud S., Ann. Phys. Fr 15 (1990), p.63,

[2] Reynaud S., Heidmann A., Giacobino E., Fabre C., Progress in Optics XXX, 1 (Elsevier Science Publisher BV, 1992).

[3] Cours à l'Ecole d'été des Houches Session 53 de H.J. Kimble (p. 549) et C. Fabre, S. Reynaud (p.679) dans "Fundamental Systems in Quantum Optics" J.M. Raimond, J. Dalibard eds, North Holland, Amsterdam (1992).

[4] Gardiner C. Quantum Noise (Springer-Verlag, 1992) 
[5] SLusher R., Holberg L., Yurke B., Mertz J., Valley J., Phys. Rev. Letters 55 (1985), p.2409. Pour une revue des expériences de production d'états comprimés, voir : Kimble H.J.,Physics Reports 219 (1992), p.227

[6] Caves C., Phys Rev D26 (1982), p. 1817, Yamamoto Y. et al. Progress in Optics XXVIII, (Elsevier Science Publisher BV, 1990)

[7] Courtois J.Y., Smith A., Fabre C., Reynaud S., J. of Modern Optics 38 (1991), p. 177

[8] Schawlow A., Townes C., Phys. Rev. 112 (1958), p.1940

[9] Kolobov M., Davidovich L., Giacobino E., Fabre C., Phys. Rev A47 (1993), p. 1431

[10] Benkert C., Scully M., Bergou J., Davidovich L., Hillery M., Orszag M., Phys. Rev. A41 (1990), p. 2756

[11] Yamamoto Y., Machida S., Nilsson O., Phys. Rev. A34 (1986), p.4025

[12] Golubev Y., Sokolov I., Sov Phys. JETP 60 (1984), p.234

[13] Henry C., Coherence, amplification and quantum effects in semiconductor lasers, (Y. Yamamoto éditeur, Wiley, 1991) p.5

[14] Haken H. Laser Theory, Section VI.7 (Springer-verlag, 1984)

[15] Lax M., Physics of Quantum Electronics (P. Kelley, B. Lax éditeurs, McGraw-Hill, 1966) p. 735 ,

[16] Ritsch H., Zoller P., Gardiner C., Walls D., Phys. Rev A44 (1991), p.3361

[17] Haake F., Kolobov M., Fabre C., Giacobino E., Reynaud S., Phys. Rev. Lett. 71 (1993), p.995

[18] Richardson W., Machida S., Yamamoto Y., Phys. Rev. Letters 66 (1991), p. 2867

[19] Wang H., Freeman M., Steal D., Phys. Rev. Letters 71 (1993), p.3951, Freeman M., Wang H., Steel D., Craig R., Scifres D., Optics Letters 18 (1993), p. 379 